\title{
Appreciation to Reviewers
}

With this issue, the Journal of Behavioral Health Services \& Research (JBHS\&R) concludes its 47 th year of publication. I am very grateful to all of the authors, reviewers, and $J B H S \& R$ Editorial Board members for their exceptional contributions to this peer-reviewed publication, particularly during this COVID-19 pandemic.

Editing and publishing a scholarly peer-reviewed journal is an amazing as well as complex exertion that involves working with a critical mass of fascinating, highly creative, well-educated, and very productive assemblage of superb individuals. Certainly, the $J B H S \& R$ could not exist without the manuscript authors, the $J B H S \& R$ Editorial Board members, and the Ad Hoc External Manuscript Reviewers.

At this time, I would like to recognize and express my profound appreciation to the many individuals who continue to support this scholarly publishing initiative: Mr. Chuck Ingoglia, Mr. Neal Comstock, and Ms. Mary Johnson at the National Council for Behavioral Health; Ms. Katherine Chabalko, Mr. Bob Darnowsky, Mr. Arene Ligo, Mr. Genesis Obero, Ms. Paola Yuli, and Ms. Sara Yanny-Tillar at Springer publishing; Dr. Mario Hernandez and Ms. Sarah Sheffield at the University of South Florida; and the academic and institutional libraries who have electronic access to the $J B H S \& R$.

This scholarly publication is committed to publishing applied services research, services delivery, policy, and implementation sciences manuscripts in behavioral health (including alcohol, drug abuse, and mental health) services. The ability to improve the quality of the manuscripts published in the $J B H S \& R$ is largely dependent upon the strength of the double-blind peer-review process. Manuscript referees provide the $J B H S \& R$ with an immeasurable wealth of expertise and willingness to volunteer their time and vivacity on behalf of the $J B H S \& R$. Therefore, I especially would like to thank and express my deepest appreciation to the individuals listed below, all of whom devotedly reviewed at least one manuscript submitted to the JBHS\&R during 2019-2020.

Bruce Lubotsky Levin

Editor-in-Chief

\author{
Martha Aby \\ Andrea Acevedo \\ Alexandra Albizu-Jacob \\ Pierre K Alexandre \\ Paige Alitz \\ Jeffrey A. Anderson \\ Denis Antoine \\ Cynthia L. Arfken
}

Dan Aune
Julie Baldwin
William Bane
Emily Baton
Rachel Battaglia
Christy L. Beaudin
Astrid Beigel
Vivian Bell

\author{
Anne H. Berman \\ Casey Bohrman \\ Christopher Bory \\ P. Qasimah Boston \\ A. Suzanne Boyd \\ Jason E. Boye \\ Jordan Braciszewski \\ Eileen Brennan
}


Lauren Brookman-Frazee

Eric J. Bruns

Jeff Capobianco

Carol Carstens

Melody Chavez

Beomyoung Cho

Johnson Chun-Sing Cheung

Michael Clarkson-Hendrix

Mariah Coe

Sara Coffey

Janet Marie Coffman

Joseph Comaty

Togas Constantinos

Alexander Cowell

Lee A. Crandall

Raina Croff

Gracelyn Cruden

Janet Cummings

James J. Dahl

Tyra Dark

Tatiana Davidson

Margot Davis

Bruce R. DeForge

Loretta L. Denering

Megan F. Dickson

Ann Dirks-Linhorst

Suzanne Dolwick Grieb

Erica Eliason

Noe Erazo

Richard A. Epstein

Ekom Essien

Joelle Ferron

Marie-Josee Fleury

Rebecca Ford-Paz

T. Freeman Gerhardt

Garcia Colato

Melissa M Garrido

Brent Gibbons

Paul B. Gold

Pnina Goldfarb

Amy L. Green

Keri J. Griffin

James Griffith

Mason Haber

Samantha M. Hack

Ardis Hanson

Eric R. Hardiman
Jeffrey Harman

Kirk Heilbrun

Linda Henderson-Smith

Sharon Hodges

Kim Hoffman

Cheryl Holm-Hansen

Cole Hooley

Laura Hume

Heidi Israel

Emily Jones

Jody Kamon

Brian Kaskie

Cheryl A. Kassed

Lourah Kelly

Suzanne Kerns

Vanessa V. Klodnick

Derrick Kranke

Ashok Kumbamu

Marsha Langer Ellison

Thomas LaPorte

Nadine Lariviere

Mary Jo Larson

Kelin Li

Alisa Lincoln

Humberto López Castillo

Amy Loree

Marilyn D. Lovett

Robert Lucio

Ami Lynch

Vickie Lynn

Jennifer L. Magnabosco

JoAnne Malloy

Michael Anthony Mancini

Ronald W. Manderscheid

Joe Marrone

Rick Massatti

Jason Matejkowski

Dennis McCarty

Roxann McNeish

Amy Mendenhall

Christopher G. Mitchell

Charles W. Mueller

Lisa Mueller

Debra A. Murphy

Katherine Nelson

Megan O'Brien

Trina Orimoto
Princess E. Osei-Bonsu

Teresa Ostler

Anne E. Pidano

Vierne Placide

Laurie Powers

Jonathan D Prince

Scott E. Provost

Michael Pullmann

Jonathan Purtle

Jason Raines

Sharon Reif

Sandra G. Resnick

Traci R. Rieckmann

David Salkever

Deborah Scharf

Susan L. Schoppelrey

Claudia Sellmaier

Sandro Serpa

Amanda Sharp

Sarah K Sheffield

Prabin Shrestha

Melissa E. Smith

Shelley A. Steenrod

Bradley Steinfeld

Larkin Street McReynolds

Christina Studts

Sarah Taylor

Gregory B. Teague

Judith L. Teich

Christine Timko

James M. Topolski

Tram HH Tran

Elise Trott Jaramillo

Lacey Tucker

Jennifer Vojtech

Enya Vroom

Melissa Walker

Neal Wallace

Weijun Wang

Ming Wang

Min Wang

Wendy Ward

Kenric B. Ware

Christine M. Walrath-Greene

Alia Renae Warner

Heidi C. Waters

Dennis Watson 
Tammy T. Webb

Amanda N. Weston

Sha-Lai Williams

Amy Wilson
Yin-Ling Irene Wong

Svetlana Yampolskaya

Anna Zamora-Kapoor

Amanda Zavodnick
Hui Zhang

Samuel H. Zuvekas

Publisher's Note Springer Nature remains neutral with regard to jurisdictional claims in published maps and institutional affiliations. 